Meyer, Anette

Die Hochschulbibliographie der Technischen Universität Ilmenau - erste Nutzerbefragung 2014 nach neun Jahren Echtzeitbetrieb

Original erschienen in:

Bibliotheksdienst. - Berlin : de Gruyter. - Bd. 50.2016, 3/4, S. 371-385.

Erschienen im Druck: 01.03.2016

Online erschienen: $\quad 15.03 .2016$

ISSN (print): $\quad$ 0006-1972

ISSN (online): $\quad$ 2194-9646

DOI:

$10.1515 / \mathrm{bd}-2016-0036$

[Gesehen

13.08.2018]

This work is licensed under a Creative Commons

Attribution-NonCommercial-NoDerivatives 4.0

International License.

(CC BY-NC-ND 4.0).

https://creativecommons.org/licenses/by-nc-nd/4.0/ 
Anette Meyer

\section{Die Hochschulbibliographie der Technischen Universität Ilmenau - erste Nutzerbefragung 2014 nach neun Jahren Echtzeitbetrieb}

DOI 10.1515/bd-2016-0036

Zusammenfassung: Die Befragung erfolgte in Form eines fragebogengeleiteten Interviews. Dazu wurden 35 Angehörige verschiedener Personengruppen aus der TU Ilmenau interviewt. Die Befragten waren mit dem Service der Hochschulbibliographie sehr zufrieden bzw. erachteten ihn als selbstverständlich. Der Anspruch an die Güte des Dienstes war sehr hoch. Probleme, die seitens der Bibliothek erwartet wurden, bestätigten sich nicht. Der Notwendigkeit, mehr Dokumente oder Objekte zu verzeichnen, wie Forschungsdaten oder Videodokumente, wurde eine hohe Wichtung beigemessen.

Schlüsselwörter: Hochschulbibliographie, Nutzerbefragung, Nutzerzufriedenheit, Datenbankinhalt, Datenausgabe

The university bibliography of the Ilmenau University of Technology - 2014: first user survey after nine years of real-time operation

Abstract: The survey was conducted as a series of interviews based on a questionnaire. 35 members of different groups of the TU Ilmenau were interviewed. The persons questioned were very satisfied with the university bibliography service or took it for granted. The demand on the service quality was very great. Problems expected by the library were not confirmed. The necessity to list more documents or objects like research data or video documents was deemed very important.

Keywords: university bibliography, user survey, user satisfaction, data bank content, data output

Anette Meyer: anette.meyer@tu-ilmenau.de 


\section{Ziel der Nutzerbefragung}

Die Hochschulbibliographie (HSB) der Technischen Universität (TU) Ilmenau ist seit $2005^{1}$ ein wichtiges Instrument für Recherche, Evaluation und Präsentation universitärer Veröffentlichungen. Nach nunmehr neun Jahren, in denen stetig und situativ Anpassungen erfolgten, sollte nun grundsätzlich untersucht werden:

- Welche Ansprüche haben die Nutzer?

- Welche Services haben an Relevanz verloren? Welche Services könnten neu aufgenommen werden?

- Sollten Arbeitsabläufe neu gestaltet werden und gegebenenfalls welche?

- $\mathrm{Zu}$ welchen Bereichen der Universität könnten neue Verknüpfungen aufgebaut werden?

Als Ziel wurde formuliert, die Hochschulbibliographie auf die Anforderungen der nächsten Jahre vorzubereiten.

\section{Vorgehensweise}

Die Wahl fiel auf die Form Befragung. Dazu wurde ein Fragebogen in einer Gruppe von vier Mitarbeitern erstellt. Rasch wurde klar, dass in den Fragen, zum Teil offensichtlich, die Meinung des Fragenden transportiert wurde - ob nun zu positiv betrachtet oder zu kritisch. Daraufhin wurden die Fragen erneut umformuliert. Der fertige Fragebogen ${ }^{2}$ wurde einer größeren Mitarbeiterrunde zur kritischen Begutachtung vorgestellt und nochmals leicht angepasst. Abbildung 1 zeigt die Fragenkomplexe.

1 Vogt, Gerhard; Meyer, Anette: Die Hochschulbibliographie der Technischen Universität Ilmenau. In: Bibliotheksdienst 40 (2006) S. 588-597.

2 Stand: Nutzerbefragung der Universitätsbibliothek Ilmenau zur Hochschulbibliographie 2014 - Fragebogen. Stand: 06.03.2014. http://www.db-thueringen.de/servlets/DocumentServlet? id=26271 [Zugriff: 31.07 .2015$]$. 
1 Ziel der Nutzerbefragung

2 Nutzung der Daten aus der Hochschulbibliographie

3 Inhalte

4 Sichtbarkeit

5 Meldungen/Formulare

6 Nachweis/Verfügbarkeit

7 Studienabschlussarbeiten

8 Ausgabe („dynamisch“): Publikationslisten

9 Ausgabe (,statisch“): Evaluationszahlen, Ausgaben mit bibliographischen Angaben

10 Schlussfrage

Abb. 1: Übersicht über die Fragenkomplexe.

Der Fragebogen wurde während des Interviews ausgefüllt im Sinne einer „Expertenbefragung “. Sie dauerte in der Regel 90 Minuten. Der Vorteil dieser Form war, dass die Fragen erläutert und Fehlinterpretationen seitens der Befragten sofort bereinigt werden konnten. Ein weiterer Vorteil war, dass die Anmerkungen, die um die Fragenkomplexe herum ausgesprochen wurden, als Kommentare mitverzeichnet werden konnten.

Die Befragungen fanden im Frühjahr/Sommer 2014 statt. Die Hochschulöffentlichkeit war auf die Befragung vorbereitet, da dies in der „Ziel- und Leistungsvereinbarung für den Zeitraum 2012 bis 2015 zwischen dem Thüringer Ministerium für Bildung, Wissenschaft und Kultur und der Technischen Universität Ilmenau“ 3 veröffentlicht, sowie im Senatsausschuss für Forschung und wissenschaftlichen Nachwuchs im Juni 2013 angekündigt und in weiteren Hochschulgremien diskutiert worden war. Dies erleichterte die Terminvereinbarung bei den Professoren wesentlich. Der Fragebogen wurde jeweils nach der Terminvereinbarung vorab verschickt.

Insgesamt wurden 35 Personen interviewt: fünf Studenten (S), zehn Professoren $(\mathrm{P})$ - das sind ca. zehn \% des Professoriums, acht wissenschaftliche Mitarbeiter (W), sieben verwaltungsnahe Mitarbeiter (V) und fünf Mitarbeiter aus der Bibliothek (UB). Dabei wurde darauf geachtet, dass diese verschiedenen Fakultäten angehörten. Mehr Personen zu befragen, wäre sicher wünschenswert gewesen, aber die Vorbereitung auf die Interviews, die Durchführung und die Nacharbeiten waren so zeitintensiv, dass eine Beschränkung erfolgen musste.

3 Abrufbar unter: www.thueringen.de/mam/th2/tmbwk/wissenschaft/hochschulentwicklung/ zlv-2012-2015-tui.pdf in der Endfassung vom 19.12.2013 [Zugriff: 21.07.2015]. 
Bei den Antworten wurde nach Wichtungen gefragt. Es konnte jeweils ein Wert auf einer Skala zwischen 1 - für unwichtig und 5 - für sehr wichtig gewählt werden. Je nach Frage wurde „kenne ich nicht“ bzw. „nutze ich nicht“ ebenfalls als Antwortmöglichkeit vorgesehen. Beispielhaft siehe dafür Abbildung 2.

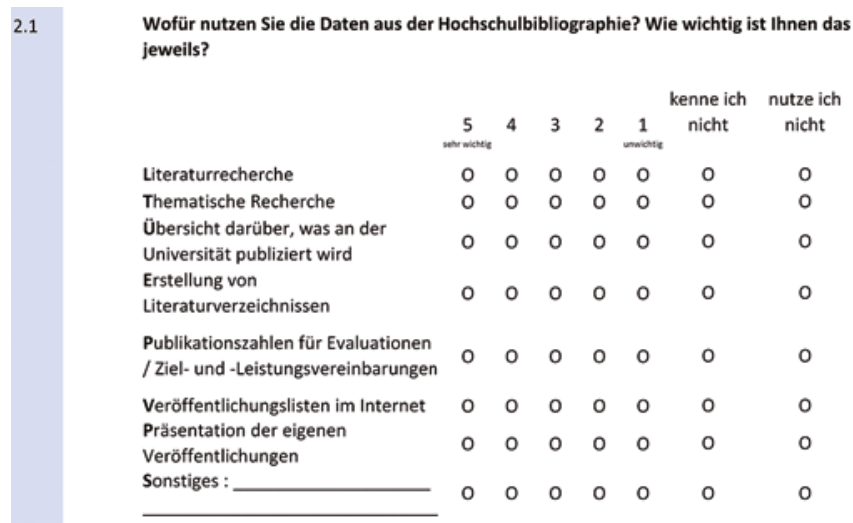

Abb. 2: Exemplarische Ansicht einer Frage.

\section{Auswertung}

Die Auswertung erfolgte mit Hilfe von Excel. Dabei erfolgte die Übertragung der Daten so, dass nicht nur die Gesamtheit der Befragten, sondern, trotz der kleinen Stichprobe, auch die Antworten bezogen auf die unterschiedlichen Personengruppen betrachtet werden konnte.

\subsection{Nutzung der Daten}

Die erste inhaltliche Frage zielte auf die Nutzung der Daten aus der Hochschulbibliographie. - „Wofür nutzen Sie die Daten aus der Hochschulbibliographie? Wie wichtig ist Ihnen das jeweils?" Bei der Auswertung musste getrennt werden: Zum einen in die Menge, welche die Möglichkeiten der Nutzung bzw. die Nachnutzung der Daten kannten, und die andere, welche sie nicht kannten bzw. nicht nutzten.

Es gab keinen Bereich, der nicht genutzt wurde (siehe Abbildung 3). Die Hauptnutzung war die generelle „Übersicht darüber, was an der Universität publiziert wurde“ und passend zu den Personengruppen die „Zahlen für die Evaluierung bzw. Ziel- und Leistungsvereinbarung“. Bei der Frage nach der Nut- 
zungshäufigkeit gaben Studenten und wissenschaftliche Mitarbeiter an, dies wöchentlich bis monatlich $\mathrm{zu}$ tun. In den Kommentaren fiel häufig das Wort „Kontrolle“. Kontrolle, ob alle Daten gemeldet wurden, Kontrolle, ob die Zuordnungen korrekt erfolgten. Hintergrund dazu ist, dass es an der TU Ilmenau eine leistungsorientierte Mittelverteilung gibt, deren Grundlage u. a. Daten der Hochschulbibliographie sind. Aber auch für die selbst gepflegten Literaturlisten und für die Literaturverzeichnisse wurde die Hochschulbibliographie als Lieferant korrekter Angaben genannt.

\section{Nutzung der Daten für...}

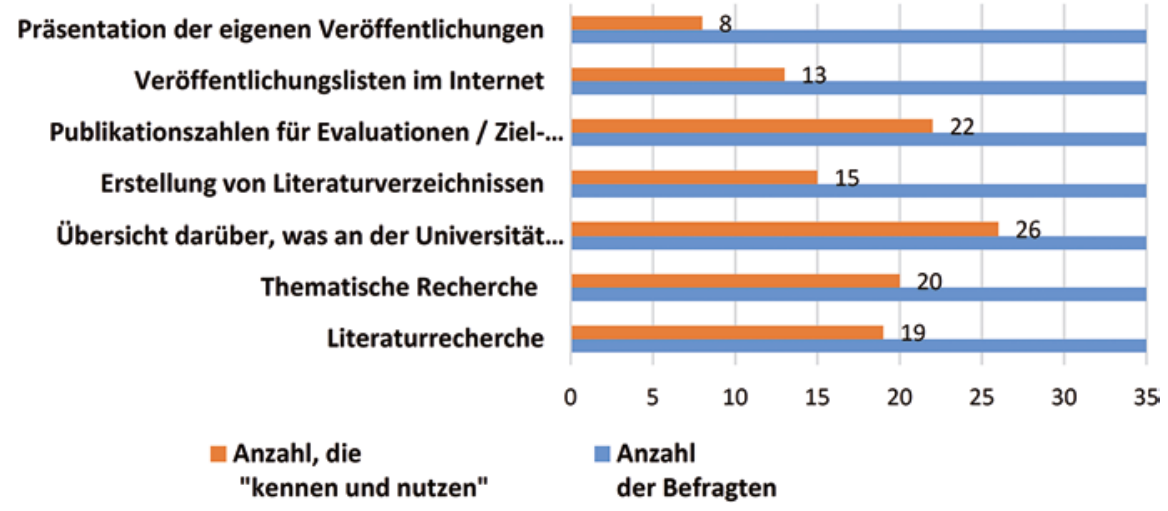

Abb. 3: Nutzung der Daten aus der Hochschulbibliographie.

Wie erwartet, lagen die Nutzungsschwerpunkte je nach „Gruppenzugehörigkeit“ unterschiedlich. Bei Betrachtung der Wichtung der einzelnen Nutzungen (siehe Abbildung 3) lagen diese für alle Personengruppen (G) zwischen 3 (mittel) und 4 (wichtig). Jedoch war die Streuung innerhalb der Nennungen hoch.

Wird die Wichtung bei den einzelnen Nutzungen nach Personengruppen getrennt betrachtet, fällt auf, dass die Studenten die thematische Recherche und die Übersicht ebenso wie die Nachnutzung der Veröffentlichungslisten überdurchschnittlich wichtig bewerteten. Die befragten Professoren schätzten die Wertigkeit bei den Veröffentlichungslisten und der Präsentation der eigenen Veröffentlichungen unter dem Durchschnitt ein. Die wissenschaftlichen Mitarbeiter (W) hingegen bewerteten gerade diese, sowie die Nachnutzung der Publikationszahlen und auch die Hilfe bei der Erstellung von Literaturverzeichnissen überdurchschnittlich. Für die Mitarbeiter der Bibliothek (UB) hatten diese keine Bedeutung. 


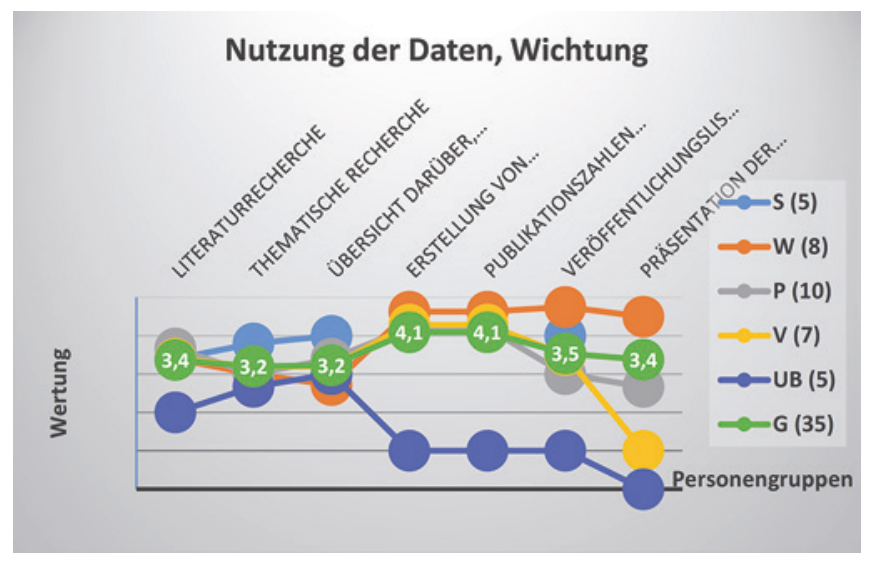

Abb. 4: Wichtung der einzelnen Nutzungen der Daten aus der Hochschulbibliographie.

Warum die Literaturrecherche diese Wertschätzung erhalten hat, bleibt offen. Eine Erklärung dazu wäre möglicherweise die unscharfe Trennung zwischen thematischer Recherche und Literaturrecherche bei der Wortwahl oder auch bei der tatsächlichen Suche im Katalog. Die Schwierigkeit, hier zu unterscheiden, zeigte sich schon in den Interviews, so dass wir hier anscheinend eine „bibliothekarische" Frage gestellt haben.

\subsection{Inhalte}

„Wie wichtig ist Ihnen, dass die folgenden Dokumente in der Hochschulbibliographie erfasst werden?" lautete die Frage. Das Ergebnis legt dar, dass wir generell mit den derzeitig erfassten Dokumenttypen immer noch richtig liegen. In den Kommentaren wurde mehrfach der Wunsch oder auch die Erwartung der vollständigen Abbildung der Publikationsleistung der Universität bzw. der Fakultät, des Instituts oder Fachgebietes geäußert. Zieht man für die Auswertung die Modalwerte (Wert, welcher am häufigsten genannt wurde) heran, so erhalten alle Dokumenttypen über alle Personengruppen gesehen, den Wert 5 (sehr wichtig, siehe Tabelle 1).

Die Betrachtung wird differenzierter, wenn man die unterschiedlichen Personengruppen z. B. die der Professoren (P), betrachtet. Bachelor-Arbeiten, Tagungsbeiträge-Abstracts, Rezensionen und Forschungsberichte erhielten von diesen am häufigsten die Wichtung 1 (unwichtig). Diplom- und Masterarbeiten wurden hingegen von den Professoren stärker gewichtet als von den Bibliotheksmitarbeitern (UB). 
Tab. 1: Dokumententypen - Berechnung der Modalwerte - Einfärbung der Modalwerte 4 und 5 (sehr wichtig).

\begin{tabular}{lcccccc}
\hline Dokumententyp & S (5) & W (8) & P (10) & V (7) & UB (5) & G (35) \\
\hline Bachelor-Arbeiten & 5 & 5 & 1 & 5 & 3 & 5 \\
\hline Bücher (Monographien) & 5 & 5 & 5 & 5 & 5 & 5 \\
\hline Buchbeiträge & 5 & 5 & 5 & 5 & 5 & 5 \\
\hline Diplomarbeiten & 5 & 5 & 5 & 5 & 3 & 5 \\
\hline Dissertationen & 5 & 5 & 5 & 5 & 5 & 5 \\
\hline Forschungsberichte & 5 & 5 & 1 & 5 & 5 & 5 \\
\hline Habilitationsschriften & 5 & 5 & 5 & 5 & 5 & 5 \\
\hline Kongressbände & 5 & 5 & 5 & 5 & 5 & 5 \\
\hline Kongressbeiträge & 5 & 5 & 5 & 5 & 5 & 5 \\
\hline Tagungsbeiträge-Abstracts & 5 & 5 & 1 & & 4 & 5 \\
\hline Masterarbeiten & 5 & 5 & 5 & 5 & 3 & 5 \\
\hline Rezensionen & 4 & 5 & 1 & & 4 & 5 \\
\hline Schriftenreihen & 5 & 5 & 5 & 5 & 5 & 5 \\
\hline Zeitschriften & 5 & 5 & 5 & 5 & 5 & 5 \\
\hline Zeitschriftenaufsätze & 5 & 5 & 5 & 5 & 5 & 5 \\
\hline
\end{tabular}

Zieht man zum Vergleich die Mittelwerte (in Tabelle 2) heran, liegt kein Wert unter 2,5 in der Wichtung. Die Gruppenunterschiede bleiben bestehen, stellen sich aber für eine Interpretation im Vergleich zu den Modalwerten, doch wesentlich abgeschwächter dar. 
Tab. 2: Dokumententyp - Berechnung der Mittelwerte.

\begin{tabular}{lcccccc}
\hline Dokumententyp & S (5) & W (8) & $\mathbf{P ( 1 0 )}$ & V (7) & UB (5) & G (35) \\
\hline Bachelor-Arbeiten & 3,6 & 4,8 & 3 & 3,8 & 3 & 3,66 \\
\hline Bücher (Monographien) & 5 & 4,9 & 4,1 & 5 & 4,5 & 4,63 \\
\hline Buchbeiträge & 5 & 5 & 4,1 & 5 & 4,5 & 4,66 \\
\hline Diplomarbeiten & 4,4 & 5 & 3,2 & 3,8 & 3,3 & 3,94 \\
\hline Dissertationen & 5 & 5 & 4,2 & 4,2 & 4,3 & 4,53 \\
\hline Forschungsberichte & 4,8 & 3,7 & 2,8 & 4 & 4,3 & 3,7 \\
\hline Habilitationsschriften & 5 & 5 & 4,2 & 4,2 & 4,5 & 4,56 \\
\hline Kongressbände & 4,6 & 4,9 & 3,7 & 5 & 4,8 & 4,47 \\
\hline Kongressbeiträge & 4,6 & 5 & 3,9 & 5 & 4,8 & 4,56 \\
\hline Tagungsbeiträge-Abstracts & 4,2 & 4,3 & 2,5 & 3,3 & 4,5 & 3,58 \\
\hline Masterarbeiten & 5 & 5 & 3,2 & 4,2 & 3,3 & 4,09 \\
\hline Rezensionen & 4 & 3,4 & 2,5 & 2,7 & 4 & 3,2 \\
\hline Schriftenreihen & 4,8 & 4,4 & 4,1 & 4,8 & 5 & 4,5 \\
\hline Zeitschriften & 5 & 4,9 & 4,1 & 4,8 & 5 & 4,66 \\
\hline Zeitschriftenaufsätze & 5 & 5 & 4,1 & 5 & 5 & 4,72 \\
\hline
\end{tabular}

Der Frage nach dem derzeitigen Ist-Stand folgte die Gegenfrage: „Gibt es Dokumente, die Ihrer Meinung nach nicht erfasst und verzeichnet werden sollten? Welche?" Aus dem Professorium gab es zwei Stimmen, welche die Bachelor-, Master- und Diplomarbeiten nicht verzeichnet sehen wollten. Gleichzeitig erfolgte aber auch die Einschränkung, dass es den Studenten frei stehe, ihre Arbeiten auf Internetplattformen zu veröffentlichen, unabhängig von deren Qualität bzw. Benotung. Zugleich kann so indirekt die Forschungsausrichtung des Fachgebietes schon in einem sehr frühen Stadium sichtbar werden. Ebenfalls wurde moniert, dass der Dokumententyp Tagungsbeiträge-Abstracts überhand nehmen würden. Vorherrschend lautete jedoch die Meinung: Lieber mehr verzeichnen als zu wenig.

Die Frage nach den Inhalten, ob der Inhalt erweitert, zusätzliche Dokumente/Daten erfasst werden sollten, wurde im Gespräch dahingehend konkretisiert, dass dies nicht nur im Rahmen der Hochschulbibliographie zu sehen sei und auch die Bibliothek nicht die ausführende Einrichtung sein müsse. Die Antworten zu diesen Fragen waren geprägt von dem Sparzwang, dem sich alle unterworfen fühlen. 


\section{Im Einzelnen:}

- Adressdaten: Diese waren den meisten nicht wichtig.

- Bilder: Schon in den ersten Sätzen wurde das Problem des Copyrights genannt oder es gab die Meinung, genügend lägen frei verfügbar im Netz. Die Verzeichnung von Bildern in den Datenbanken des Universitätsarchivs und des Referats Marketing war einigen bekannt und wurde als ausreichend erachtet.

- Forschungsdaten: Hier wurde die Bedeutung einer sichtbaren bzw. öffentlichen Verzeichnung gesehen - und dass hier Handlungsbedarf bestehe.

- Objekte/Sammlungen: Sofort wurde der Aufwand der Verzeichnung bzw. Beschreibung der Objekte angeführt. Jedoch bedauerten auch einige, dass Sammlungen verloren gegangen sind. Bei den Neuausrichtungen von Fachgebieten fanden diese keine fortfolgende Wertschätzung und wurden entsorgt.

- Softwaredateien: Die Meinungen reichten hier von „Pixelgrab“ bis dahin, dass es wichtig sei, auch außerhalb der Foundations (wie gitHub, sourceForge) einen Archivierungsort der Quellcodes zu haben.

- Tondokumente: Wer sammelt Tondateien oder die eigenen auch aktuellen Zeitdokumente wie Rundfunkreportagen? Wo archiviert der von den Studenten betriebene Hochschulfunk?

- Videodokumente: Wer archiviert? Für die Verbreitung wichtig ist „YouTube“, aber wo ist z. B. der von Prof. Horst Zuse im Jahr 2011 gehaltene Festvortrag zur Einweihung des Zusebaus wiederzufinden? Es gäbe bereits auch etablierte Plattformen. Genannt wurde die „Gallery of Fluid Mechanics“ (umbenannte in „Gallery of Fluid Motions“.) Es gibt Beiträge in Videoform, die bei Kongressen eingereicht werden (z. B. http://gfm.aps.org/). Die Videos dort sind mit DOIs versehen, aber wie sind diese recherchierbar? Oder es gibt in Zeitschriften wie im „New Journal of Physics“ Videobeiträge, die online betrachtet werden können?

- Sonstiges: Hier gab es fünf Nennungen, welche die Wichtung 3 bis 5 erhielten. Genannt wurden Patente, Muster, Vorlesungsskripte, Rankings, Preise, Graphical Abstracts oder auch Events.

Welche Konsequenz soll, will oder kann die UB aus dem Ergebnis (siehe Tabelle 3) ziehen? Die Erweiterung der verzeichneten Daten in der Hochschulbibliographie z. B. auf Videodokumente oder Forschungsdaten verlangt nicht nur die Einbeziehung anderer Metadaten. Dem Verzeichnen folgt sofort die Anforderung auf Wiederauffindbarkeit und dauerhafte Zugriffsmöglichkeit. Dazu sind entsprechende Archivserver und Sicherungssysteme sowie das Knowhow, solche zu betreiben notwendig oder das Geld, diesen Service einzukaufen. 
Tab. 3: Erfassung zusätzlicher Daten.

\begin{tabular}{|c|c|c|c|c|c|c|c|c|}
\hline Bedeutung zusätzlicher Erfassung & 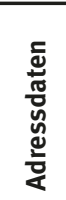 & $\frac{\bar{d}}{\frac{\overline{0}}{0}}$ & 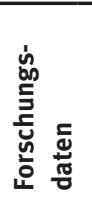 & 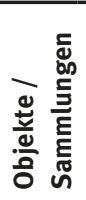 & 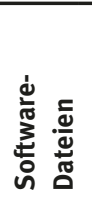 & 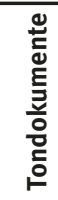 & 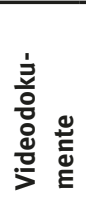 & 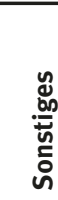 \\
\hline Prozent-Anteil mit Wichtung $4-5$ & 9 & 27 & 59 & 47 & 57 & 45 & 59 & 80 \\
\hline Prozent-Anteil mit Wichtung 3-5 & 21 & 53 & 75 & 67 & 71 & 69 & 83 & 100 \\
\hline
\end{tabular}

Zur Frage nach den Inhalten, wie wichtig es sei, die unterschiedlichen Ausgaben (Papier-, elektronische, Onlineausgabe) einer Veröffentlichung zu dokumentieren, lagen die häufigsten Nennungen bei der Wichtung 5 (sehr wichtig). Die Fachgebiete haben jedoch unterschiedliche Veröffentlichungskulturen. Die Statements lauteten von „Gedruckt ist ein Muss und das Klassische“ bis zur Aussage „Wir haben nichts Gedrucktes“. Verzeichnet werden soll offensichtlich alles. Ein sehr treffender Kommentar lautete: „Denn wenn es nicht verzeichnet ist, weiß ich auch nicht, dass es das sonst noch gibt.“

Auf die Frage: „Falls nur eine Ausgabe der Veröffentlichung verzeichnet wird, welche sollte es sein?" sprachen sich die meisten, interessanterweise aber nur zwei Drittel (in 24 von 34 Nennungen), für die Onlineausgabe aus. Wobei dazu die Rückfrage notwendig war, wie sie denn gerne selbst an die Veröffentlichungen kommen möchten. „Je nach Bestand“ oder „die gedruckte Ausgabe“ wählten je fünf Befragte. Einzelne Kommentare lauteten: Die Papierausgabe für Bücher, die Onlineausgabe für Zeitschriften, oder dass Onlinedokumente veränderbar oder sogar flüchtig seien.

\subsection{Sichtbarkeit}

Wir erfragten, ob die Hochschulbibliographie, die ja Bestandteil des Katalogs ist und auch als separater Katalog angeboten wird, schon einmal genutzt wurde. Dies hatten ca. 50 Prozent der Befragten getan. D. h. 50 Prozent hatten sie bisher nicht genutzt, wovon ein Drittel nicht wusste, dass es diese überhaupt separat gibt. Dass sie vier der fünf Studenten nicht kannten, war zu erwarten, aber bei den Professoren und wissenschaftlichen Mitarbeitern kam diese Antwort dann doch überraschend. Denn die UB informiert einmal im Jahr zum Jahresende die Fachgebiete (die E-Mail wird über die Dekanate verschickt) über die in der Hochschulbibliographie verzeichneten Titel - mit entsprechenden Links. 
Darauf folgte die Frage: „Die Hochschulbibliographie ist Bestandteil des Ilmenauer Katalogs. Wie finden Sie das?" Hier baten wir, die Auswahl zu begründen.

„Überwiegend gut - weil...“ nur ein Katalog genutzt werden muss und man alle Daten sehen möchte. Ähnliche Aussagen finden sich auch in den Kommentaren wieder wie: „Bei der Suche findet man zumindest etwas.“ „Es ist frustrierend, wenn man nichts findet.“

„Gut - weil...“ „Wer, wenn nicht die eigene Universität sollte die Publikationen im Katalog verzeichnen?“

„Nicht gut - weil...“ Die Mitarbeiter der UB, die an der Befragung teilgenommenen hatten, fanden dies nicht gut. Die Meinungen auf die Gruppen aufgeteilt, sind in Abbildung $5 \mathrm{zu}$ sehen.

\section{Hochschulbibliographie als Teil des Kataloges}

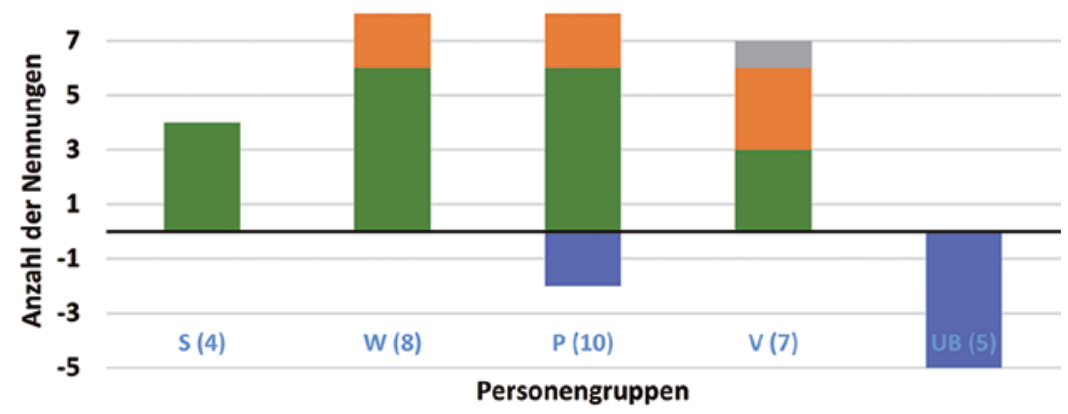

Eut, weil $=$ Ist mir noch nicht aufgefallen $=$ Dazu habe ich keine Meinung $\equiv$ Nicht gut, weil

Abb. 5: Meinungsbild zur Hochschulbibliographie als Teil des Kataloges.

\subsection{Meldungen/Formulare}

Von den 35 Befragten hatten 19 schon einmal selbst ihre Veröffentlichung gemeldet. Die Meinung war, dass es überwiegend leicht sei, mit der „Hochschulbibliographie“ zu kommunizieren, ob über die Formulare per E-Mail oder über sonstige Wege. Es kamen einzelne Verbesserungsvorschläge zum Vereinfachen der Meldeformulare, die wir gleich umsetzen konnten. 


\subsection{Nachweis/Verfügbarkeit}

Auf die Frage: „Wie finden Sie es, dass die Hochschulbibliographie auch Publikationen nachweist, die in der Universitätsbibliothek nicht physisch vorhanden sind bzw. auf die nicht unmittelbar zugegriffen werden kann (z. B. Zeitschriftenaufsätze, die weder in Papierform noch als Online-Zugriff vorhanden sind)?“ fanden dies alle „sehr gut“. Hier wiederholte sich der Wunsch nach vollständiger Verzeichnung, unabhängig von der Verfügbarkeit vor Ort.

Die gleich nachfolgende Frage: „Wären Sie bereit, Ihre Dokumente open access zur Verfügung zu stellen?“, wurde auch mit einem einhelligen Ja beantwortet, welchem ein sofortiges Aber folgte. Es herrschte große Unsicherheit, ob und in welchem Ausmaß die derzeitige Rechtslage es zulässt, die eigenen Publikationen nachzuveröffentlichen. Auch wurde das Ansehen der „Open-AccessZeitschriften“ kritisch gesehen.

Hier kann nur mehr erreicht werden, wenn seitens der UB aktiv beraten wird. Aber können die Beratungen rechtssicher über alle Verträge und Gesetze hinweg erfolgen? Der Open-Access-Gedanke wird an der TU Ilmenau durch eine verabschiedete Open-Access-Policy ${ }^{4}$ begleitet und durch einen 2015 eingerichteten Publikationsfonds untersetzt. Offensichtlich bedarf es jedoch stetiger Beratung und Bewerbung, welche u. a. durch die Benennung eines an der Bibliothek angesiedelten Open-Access-Beauftragten befördert wird.

\subsection{Studienabschlussarbeiten}

Die Diplom-, Master- und Bachelorarbeiten werden seit dem Jahr 2006 in der Hochschulbibliographie nachgewiesen. Neben den obligatorischen Titeldaten werden Betreuer und Gutachter verzeichnet. Ein echter Mehrwert wird durch die gleichzeitige Eintragung einer im Katalog durchsuchbaren Zusammenfassung erzielt. Jedes Jahr sind dies ca. 1500 Titel, häufig ohne dass die Bibliothek diese bereitstellen könnte. Verzeichnet wird auf Grundlage der „Allgemeinen Bestimmungen“5 der Studienabschlüsse Diplom, Master oder Bachelor.

\footnotetext{
4 Erklärung der Technischen Universität Ilmenau über den freien Zugang zu wissenschaftlichen Forschungsergebnissen. Beschlossen durch den Senatsausschuss für Forschung und wissenschaftlichen Nachwuchs am 11. März 2014 und bestätigt durch den Senat der Technischen Universität Ilmenau am 15. April 2014. http://www.tu-ilmenau.de/fileadmin/media/ub/div/OA-ErklaerungTU_Ilmenau.pdf [Zugriff: 21.07.2015].

5 Beispiel: „§12 (8) Mit der Abgabe der Master-Arbeit ist gleichzeitig eine kurze Zusammenfassung (Abstract) in deutscher und englischer Sprache für den Zweck der Veröffentlichung an-
} 
Gefragt wurde: „Nutzen Sie in irgendeiner Form diese Informationen zu den Studienabschlussarbeiten?“ Die Hälfte der Befragten nutzte diese zu Recherchezwecken. Mit unterschiedlicher Intention: Zur Übersicht, welche Arbeiten im Fachgebiet bearbeitet wurden, ob die Thematik schon erarbeitet wurde, welche Arbeiten abgeschlossen wurden, bei wem man zur Einsichtnahme nachfragen kann. Positiv wurde erwähnt, dass es keine Ausnahmen gibt und auch bei Arbeiten, die in Kooperation mit der Industrie entstanden, die Zusammenfassungen veröffentlicht sind.

Von den 18 befragten Professoren und wissenschaftlichen Mitarbeitern nutzen 11 diese auch für ihre Veröffentlichungslisten im Internet.

Auf die explizite Frage „Stören sie im Katalog?“ (nicht in der Hochschulbibliographie) kam von den Studenten folgende, auch für andere sehr bezeichnende Antwort: „Sie stören mich eigentlich schon, aber nicht so, dass ich den Drang hätte, dafür eine Lösung zu finden.“ Andere kannten die „Hilfen“ auf der Katalogstartseite und die Möglichkeit, die Abschlussarbeiten herauszufiltern.

Von den Abschlussarbeiten sollten mehr online verfügbar sein, antworteten 26 von 34 Befragten. Gerade die Studenten würden gerne die Arbeiten - allerdings die der anderen - lesen.

\subsection{Ausgabe („dynamisch“): Publikationslisten}

Aus dem Katalog bzw. der Hochschulbibliographie können Daten ausgelesen und aufbereitet werden, so dass diese in Form von Listen in die Internetauftritte z. B. der Fachgebiete der TU Ilmenau eingebunden werden können. Zurzeit werden für 68 Struktureinheiten 117 Listen erzeugt. (18 Gesamtlisten, 42 Listen mit Publikationen, 48 Listen mit Studienabschlussarbeiten sowie 9 besondere Listen).

Der Zielgruppe Professoren und wissenschaftliche Mitarbeiter war der Service überwiegend bekannt, aber leider nicht zu 100 Prozent. Von den acht, welche den Service nicht ausreichend fanden, konnten sich nur zwei hierzu eine finanzielle Unterstützung der Bibliothek vorstellen.

zufertigen und in elektronischer Form abzugeben. Die Universität kann die Abgabe in einem bestimmten elektronischen Format vorschreiben und hierzu nähere Regelungen festlegen. Sie ist berechtigt, die Ausgabe des Zeugnisses von der Erfüllung dieser Verpflichtung abhängig zu machen. Die Universitätsbibliothek ist berechtigt, die kurze Zusammenfassung auch ohne ausdrückliche Genehmigung des Studierenden zu veröffentlichen und verbreiten.“ Aus: Allgemeine Bestimmungen - für Studiengänge mit dem Studienabschluss „Master“. In: Verkündungsblatt der Technischen Universität Ilmenau 24 (2006), S. 17-35 . http://www.db-thueringen.de/servlets/ DocumentServlet?id=7417 [Zugriff: 21.07.2015]. 
Gefragt wurde nach den Vorstellungen zu Sortiermöglichkeiten bzw. Layoutvarianten. Hier kam der Wunsch nach einer Nachnutzbarkeit der Publikationslisten, um diese in Literaturverwaltungsprogramme einzulesen, oder nach Facettierungsmöglichkeiten der Daten. Es war nicht klar, dass wir über ein Typo3Plugin mit Hyperlinks angereicherte Listen erzeugen. Die Wünsche wären mit selbst-parametrisierbaren Datenbanken im eigenen Webauftritt zu erfüllen. Für das Facettieren konnte eine Lösung angeboten werden. Die gewünschten Ausgabemöglichkeiten finden sich in der Ursprungsdatenbank, dem Katalog oder auch im Ilmenauer Discovery Tool. Diese Möglichkeiten waren zumeist nicht bekannt.

Eine weitere Frage lautete: „Welche Aktualität der Publikationslisten ist für Sie notwendig?“ Eigentlich hätte jeder gerne die Daten sofort aktualisiert, nämlich dann, wenn er auf die Listen schaut - der Zusatz zu der Frage, dass die Server dadurch belastet werden, führte zu der überwiegenden Meinung, dass eine monatliche Aktualisierung ebenfalls ausreichend sei.

Auf die Frage: „Falls Sie Autor sind: Sollen Publikationen, welche entstanden, als Sie nicht Angehöriger der TU Ilmenau waren, verzeichnet werden?“ waren bei 28 Nennungen 64 \% der Meinung: Ja. Ist dies ein Service, den die UB stärker anbieten sollte?

\subsection{Ausgabe (,statisch"): Evaluationszahlen, Ausgaben mit bibliographischen Angaben}

Nachgefragt werden überwiegend Daten, die mit Excel nachnutzbar sind. Die Daten werden in Blätter unterteilt. Sie enthalten die gewünschten Sortiermöglichkeiten, Spalten und Layouts. Zu diesen Zuarbeiten der Bibliothek wurde nichts angemerkt. Die korrekte Anzahl der Veröffentlichungen, die korrekte Zuordnung, die korrekte Zitierung wurden einfach erwartet. Dieser sich nicht von ganz alleine generierende Service der Bibliothek wurde eher als selbstverständlich erachtet.

\subsection{Schlussfrage - Welche Anregungen hätten Sie noch für uns?}

Von den Befragten, über die Professoren bis zu den Studenten, wurde der Bibliothek insgesamt ein positives Zeugnis ausgestellt. Genannt wurden die Raumsituation, die Freundlichkeit der Mitarbeiter, die Ausstattung, die Öffnungszeiten und anderes. Das war sehr erfreulich und kann uns nur bestärken in unserem Bemühen, stabile und gute Dienstleistungen zu erhalten und auszubauen, sowie neue Services anzubieten. 


\section{Fazit}

Die Frage: „Sollen wir eine Hochschulbibliographie pflegen oder nicht?“ muss mit Ja beantwortet werden. Ihre Existenz scheint mittlerweile selbstverständlich zu sein. Ebenso herrscht über die Sichtbarkeit der verzeichneten Dokumente Einigkeit - Sichtbarkeit ist wichtig - für den Informationsaustausch innerhalb der Universität aber noch wichtiger für das Gesehen werden der Veröffentlichungs- bzw. Forschungsleistung der Universität weltweit. An die UB wird ein hoher Anspruch gestellt. Erwartet wird: Vollständige und korrekte Verzeichnung. Die UB bietet einen positiv gesehenen Dienst an, für dessen Qualität, Stabilität und Weiterentwicklung sie in die Pflicht genommen wird. Und dennoch muss die UB Präsenz zeigen in den Gremien, Fakultäten und Fachgebieten, um den Bekanntheitsgrad der Hochschulbibliographie mit ihren um sie herum angebotenen Dienstleistungen hoch zu halten.

\section{Dr. Anette Meyer}

Universitätsbibliothek Ilmenau

Postfach 100565

98684 Ilmenau

E-Mail: anette.meyer@tu-ilmenau.de 